MARCONDES, C. H.

\section{Datos abiertos enlazados de archivos, bibliotecas y museos en la web}

Barcelona: Editorial UOC, 2018

La cantidad de datos que manejan archivos, bibliotecas y museos, y su posterior volcado en la web genera una volumen de información que se hace invisible si no se encuentra la forma de recuperarla. El uso de la web nos permite recuperar los datos abiertos enlazados, LOD en sus siglas en inglés (Link Open Data) que describen un método de publicación de los datos de una forma estructurada en la que se pueden interconectar en función de la búsqueda que hacemos.

Las tecnologías que se ocupan de los datos abiertos enlazados son parte de la llamada web semántica. Una forma de representar los contenidos que encierran estas instituciones, para que puedan ser leídos y comprendidos tanto por ordenadores como por los usuarios de archivos, bibliotecas y museos.

Para explicar la evolución de los datos abiertos enlazados, Marcondes realiza un recorrido del uso de la tecnología aplicada a los datos contenidos en los catálogos de archivos, bibliotecas y museos. Revisando las principales instituciones dedicadas a la conservación y difusión de contenidos históricos. Donde destacan los ejemplos estadounidenses como la Biblioteca del Congreso; los europeos con la Biblioteca Europeana y algunos ejemplos sudamericanos extraídos de Brasil y de México. Habla de las diferentes tecnologías de la web semántica como la representación de contenidos RDF (Resource Description Framework), y de identificadores persistentes como URLX, URI, IRI, cuya función es servir de enlace entre los datos de distintas webs. Además de las licencias de uso libre de estos contenidos. A pesar de la cantidad de acrónimos y términos en inglés que se usan a lo largo de todo el texto, hay que destacar que una vez expuestas las ideas principales aparecen ejemplos muy cercanos para que puedan ser entendidos fácilmente por el lector.

Es notable el esfuerzo no solo por poner ejemplos cercanos referidos a la Wikipedia, o a ejemplos muy clarificadores en los que se usa como muestra la búsqueda que se podría hacer sobre el Quijote. Además en cada capítulo retoma alguno de los elementos nombrados en el anterior. De esta manera se hace más fácil la lectura en un libro que, a pesar de su brevedad, es prácticamente un manual de introducción a la comprensión y uso de las tecnologías de datos enlazados.

Una vez introducido al lector en los datos enlazados abiertos, comienza a profundizar en la representación de los contenidos de los archivos, bibliotecas y museos. Donde explica las dificultades de representar de una 
forma entendible estos datos para el público general. Poniendo los principales ejemplos de vocabulario de tecnologías LOD que podemos encontrar hoy en día en la web como son el DC (Dublin Core), los vocabularios de la Fundación Getty, los vocabularios de la Biblioteca del Congreso de los Estados Unidos, VIAF (Virtual International Authority File), Iniciativa SPAR, Schema.org, vocabularios de Wikipedia, BDpedia y Wikidata, VRA (Visual Resources Association), SKOS (Simple Knowledge Organization System), Spectrum, IconClass, LIDO (Lightwight Infmation Describing Objects) y tesauros de bienes culturales del Ministerio de Cultura de España.

Siempre hace aclaraciones y separa las explicaciones y funcionamiento de archivos, bibliotecas y museos, aunque en la práctica el enlace de datos que se hará será a través de los acervos documentales de estos tres tipos de instituciones y es ese precisamente uno de los objetivos del libro. Mostrar la posibilidad de enlazar los contenidos de estas instituciones, pero también la dependencia que existe en la investigación entre estas tres. Con todos los ejemplos que se proponen en esta obra, el autor llega a desarrollar un nuevo perfil profesional dentro de los archivos, bibliotecas y museos. El curador digital, una especie de documentalista especializado en la búsqueda, enlace y representación de los contenidos que los archivos, bibliotecas y museos ya han estudiado y volcado en la red. La velocidad de los cambios en el ámbito de la informática y el procesamiento de los datos, hace necesaria una continua actualización de los conocimientos y habilidades empleadas en los datos abiertos enlazados en bibliotecas, archivos y museos. Aunque paradójicamente el desarrollo de las tecnologías LOD ha llevado a que los contenidos que enlaza sea más independientes de los programas específicos que son necesarios para hacerlos visibles y legibles para nosotros. Concluye finalmente con la necesidad de desarrollar las competencias digitales de aquellos profesionales que se dedican a la información y documentación y los problemas que puede plantear el uso de licencias abiertas entre distintas instituciones y países.

Alejandro Guillén Santoro | historiador del arte

URL de la contribución <www.iaph.es/revistaph/index.php/revistaph/article/view/4562> 